\title{
Representaciones sociales sobre estilo de autoridad y tipos de interacción en cuidadores de residencias de protección infantil en Chile
}

\author{
Marcelo Andrés Gallegos-Fuentes \\ Magíster en Familia. Asistente Social \\ Universidad del Bío-Bío. Chillán, Chile \\ https://orcid.org/0000-0002-6153-5514 • mgallegos@ubiobio.cl
}

\section{Carmen Gloria Jarpa-Arriagada}

Doctora en Ciencias de la Educación. Asistente Social

Universidad del Bío-Bío. Chillán, Chile

https://orcid.org/0000-0002-9896-5649 • cjarpa@ubiobio.cl

\section{Resumen}

El artículo aborda las representaciones sociales de cuidadores en residencias de protección. El estudio se realizó bajo el enfoque de Representaciones Sociales. Los hallazgos muestran tensión entre la Doctrina del Menor en situación irregular y la de Protección Integral. Esta tensión se resuelve mediante un ejercicio de autoridad que transita desde lo autorizativo, hasta lo negligente, con predominancia de evasión en la resolución de conflictos. Explicamos los resultados como núcleos figurativos del ejercicio de autoridad. La preeminencia de los derechos ha provocado alteraciones de jerarquía que facilita la tiranía de parte de los niños. El ejercicio de autoridad se vuelve errático y oscilante entre alternativas que reproducen el enfoque de necesidades, la visión del niño como un expósito y la incipiente presencia de un relato sobre protección integral

Palabras clave: Derechos del niño; Protección de la infancia; Institucionalización; Relaciones interpersonales; Estilo de autoridad; Cuidado de infantes.

Recibido: 01/10/2020 |Aprobado: 10/11/2020 |Publicado: 01/01/2021

(c) (1)@(2) Esta obra está bajo una Licencia Creative Commons Atribución-NoComercialEY No SA Compartirlgual 4.0 Internacional.

Financiación o proveniencia del artículo: artículo derivado del Proyecto de investigación Representaciones Sociales sobre los derechos del niño y de la niña de cuidadores que se desempeñan en sistemas residenciales del Servicio Nacional de Menores de la provincia de Ñuble, código DIUBB 155824 4/I, Universidad del Bío-Bío. Chile.

\section{¿Cómo citar este artículo? / How to quote this article?}

Gallegos-Fuentes, M., y Jarpa-Arriagada, C. G. (2021). Representaciones sociales sobre estilo de autoridad y tipos de interacción en cuidadores de residencias de protección infantil en Chile. Prospectiva. Revista de Trabajo Social e intervención social, (31), 369-392. doi: 10.25100/ prts.v0i31.8821. 
Gallegos-Fuentes y Jarpa-Arriagada

\title{
Social representations about style of authority and types of interaction in caregivers of child protection residences in Chile
}

\begin{abstract}
The article addresses the social representations of caregivers in protective residences. The study was carried out under the Social Representations approach. The findings show tension between the Doctrine of the Minor in an Irregular Situation and that of Integral Protection. This tension is resolved through an exercise of authority that ranges from authoritative to negligent, with a predominance of evasion in the resolution of conflicts. We explain the results as figurative nuclei of the exercise of authority. The preeminence of rights has caused alterations of hierarchy that facilitate the tyranny of children. The exercise of authority becomes erratic and oscillates between alternatives that reproduce the needs approach, the vision of the child as a foundling and the incipient presence of a story of comprehensive protection.

Keywords: Children's rights; Child protection; Institutionalization; Interpersonal relationships; Authority style; Infant care.

Sumario: 1. Introducción, 2. Metodología, 3. Hallazgos, 3.1 Estilos de autoridad según cuidadores/as, 3.2 Tipos de interacción cuidador/a-niño/a en contexto residencial, 4 . Conclusiones, 5. Referencias bibliográficas.
\end{abstract}




\section{Introducción}

Resulta irrefutable que la Convención Internacional de los Derechos del Niño (CIDN) y su ratificación con una alta adhesión mundial, ha logrado instalar una discusión consistente sobre la Doctrina de la Protección Integral. En efecto, luego de su suscripción, en Chile se inicia un proceso de progresivos cambios, con miras a dar cumplimiento cabal a sus principios fundamentales; en este contexto, surge la necesidad de abandonar el concepto clásico de protección, desde una mirada unidireccional y adulto céntrica, hacia lógicas de sistemas de protección transversales, integrales e inclusivos. No obstante lo anterior, desmontar la Doctrina del Menor en Situación Irregular ha sido un trabajo arduo, difícil y aún pendiente. La hegemonía del modelo tutelar y la profunda adhesión a sus fundantes ideas de control, estabilidad y orden social lograron encarnarse en sistemas, instituciones, programas y personas. Bajo la lógica de este modelo había muchas certezas y todas ellas vinculadas a la necesaria intervención ante la irregularidad social, los vicios, las disfuncionalidades familiares, el adulto centrismo, el patriarcado, la filantropía, el asistencialismo, por nombrar algunas; todas ellas han formado parte de nuestra historia personal, familiar, social, cultural o institucional.

Este artículo pretende contribuir al debate y discusión del trabajo con niños, niñas y adolescentes (NNA), en el marco de instituciones de protección infantil. Indagamos en las representaciones sociales de los adultos a cargo de estos NNA: Los cuidadores/as. Nos parece relevante indagar en los universos semánticos de los cuidadores porque son los que, en primera línea, deben personificar el cambio paradigmático. Ergo, si la nueva doctrina sólo se queda en lo declarativo, la instalación de nuevos dispositivos de protección integral perderá fuerza y seguirá reproduciendo la antigua (pero añorada) Doctrina del Menor en Situación Irregular.

La Doctrina de la Situación Irregular se gesta en un periodo histórico donde el Estado desarrolla una intensa labor reguladora de la vida privada de los sujetos (S. XIX); bajo esta concepción, la crianza de los niños es entendida como una responsabilidad que recae sobre la familia pero, fundamentalmente, sobre las mujeres (Vincenzino, 2017). Ergo, el interés del Estado en esta materia descansa sobre la necesidad de formar seres humanos funcionales para el sistema, por tanto, no solo la supervivencia de los niños es fundamental, sino también (o principalmente) reducir los costos que ocasionan aquellos que puedan ser abandonados por las familias y, como fruto de lo anterior, su cuidado y crianza quede en manos del Estado. De esta manera, el Estado debe garantizar el orden social a partir de la estabilidad lograda en la vida doméstica (Vincenzino, 2017).

Así entendida, la Doctrina de la Situación Irregular consiguió una amplia adhesión sobre la base de una ideología de orden social y principal proveedora de estabilidad para todos los ciudadanos. Lamentablemente, logró asociar a los grupos carenciados o 
Gallegos-Fuentes y Jarpa-Arriagada

vulnerables con la noción de peligrosidad (Barrera-Dávila, 2014), lo que explica las acciones decididas del Estado en rescatar a todos aquellos que podrían estar expuestos a peligro moral o material. Entonces, el Estado interviene cuando las familias fallan, legitimándose el alejamiento del niño de su grupo familiar y su ingreso a instituciones "disciplinadoras" (Farías, 2003). Las consecuencias de la hegemonía de esta doctrina son patentes hasta el día de hoy, con una fuerte carga de modelos tradicionales de familia, de la idea de funcionalidad/disfuncionalidad e incluso en la reciente noción de competencias (incompetencias) parentales.

La veloz propagación de esta doctrina no tardó en vincular, indefectiblemente, la situación irregular con el menor irregular, derivando en varias consecuencias, todas ellas muy peligrosas. Primero, supeditó la comprensión de los menores a ser objetos de tutela y protección, por considerarlos incapaces e inmaduros (Barrera-Dávila, 2014); segundo, relacionó al niño irregular con conductas delincuenciales, disruptivas o amenazantes para el orden social; tercero, al concebir al menor irregular como un sujeto distinto del niño bien cuidado por una familia funcional, creó simbólicamente un otro excluido, los demás, los menores (Campos-García, 2009); tercero, se estableció todo un aparataje institucional dirigido a lograr control social de esta problemática (legislación, instituciones y juzgados especiales), nutriendo la estigmatización de estos menores irregulares; cuarto, se desvió la atención de las causas que provocan la irregularidad y se enfatizó en la atención protectora y rehabilitadora, sin desarrollar acciones preventivas (Campos-García, 2009); quinto, ignoró los derechos del niño como persona en desarrollo y ante infracciones de parte de estos menores, el juez era plenipotenciario en decidir el destino del niño, lo que establecía una discrecionalidad para las medidas punitivas a aplicar (Beloff, 2000).

Como podemos apreciar, la Doctrina de la Situación Irregular logró instalar la idea de una categoría infancia polarizada en dos extremos; aquellos niños que logran vivir en sus familias y que tienen acceso a condiciones mínimas de vida y, los que no (Campos-García, 2009). Producto de esta esencia doctrinal, se legitima una intervención estatal discrecional sobre una subcategoría de la infancia: los menores (García-Méndez, 1994). Consecuentemente, son estos menores los que producto de serias carencias estructurales y que deberían ser proveídas por el Estado, quedan catalogados como delincuentes, infractores, disfuncionales, peligrosos. Paradojalmente, no existe equivalencia en los esfuerzos del Estado por desarrollar políticas sociales, de suficiente calidad y extensión, para proveer a toda la población infantil de lo necesario para su desarrollo integral. Ciertamente, las energías están puestas en la implementación de mecanismos de control social, con una fuerte connotación punitiva (Prieto-Cruz, 2012) y en una robusta matriz asistencialista que procura una seudoprotección (Barrera-Dávila, 2014). En suma, la Doctrina de la Situación Irregular y su modelo tutelar predominante, ofrece respuestas desarticuladas, prejuiciosas e ineficientes, que se configuran sin tomar en cuenta las peculiaridades de los niños, niñas y adolescentes (Maldonado-Fuentes, 2014), estableciendo 
una relación de control social más que de desarrollo integral, cuestionando profundamente la vigencia y consideración de los derechos de los niños.

Opuestamente a lo anterior, la Protección Integral puede ser entendida como "el conjunto de acciones, políticas, planes y programas que con prioridad absoluta se dictan y ejecutan desde el Estado, con la firme participación y solidaridad de la familia y la sociedad para garantizar que todos los niños y niñas hasta los 18 años, gocen de manera efectiva y sin discriminación de los derechos humanos a la supervivencia, al desarrollo y a la participación, al tiempo que atiende las situaciones especiales en que se encuentran los niños individualmente considerados o determinado grupo de niños que han sido vulnerados en sus derechos" (Barrera-Dávila, 2014, p. 24). Entonces fija como uno de sus principios centrales el interés superior del niño, hace explícita consideración a las características propias de esta etapa vital y pretende garantizar el desarrollo de todos los niños, sin distinción (Campos-García, 2009). De manera consecuente, también encuentra sus fundamentos en los principios universales de justicia social, equidad y dignidad y en los principios especiales de solidaridad, participación, prioridad absoluta y no discriminación (Barrera-Dávila, 2014). Ciertamente, el giro sustancial de esta doctrina es proteger derechos, más que proteger personas; de esta manera, se reduce la posibilidad de estigmatización y segregación de los más vulnerables (Campos-García, 2009), estableciéndose de manera prístina que todos/as los niños/as son sujeto pleno de derechos.

Respecto del sistema de protección infanto-juvenil en Chile, su responsabilidad le corresponde al Servicio Nacional de Menores (SENAME), institución perteneciente al Ministerio de Justicia, creado en 1979. El SENAME desarrolla su labor mediante centros que administra directamente y con una red de instituciones colaboradoras, bajo el principio de la subsidiariedad del Estado. Se encarga de la protección de niños, niñas y adolescentes (NNA), hasta los 18 años. La gran mayoría de su oferta requiere de judicialización para su ingreso, permanencia y egreso. Las residencias constituyen una de las líneas programáticas, proporcionando una estadía temporal para la atención proteccional, psicológica y social a niños/as que por orden judicial han sido separados de sus familias, bajo la sospecha de un riesgo severo en la protección de sus derechos.

Las residencias pueden adoptar características distintivas según la edad de los niños/as, tipos o gravedad de la vulneración de derechos. Para todos los efectos, estos centros de protección están "declarados" como espacios de separación transitoria con el núcleo familiar más cercano. Supone una medida de protección judicial presentada ante los Tribunales de Familia. En este sentido, las residencias deben garantizar el pleno ejercicio de derechos por parte de los niños/as, así como intervención psicosocial a los propios niños/as y a sus familias con el propósito de reinsertar, en el plazo más perentorio, a los NNA a sus familias de origen o a familias sustitutas. 
Gallegos-Fuentes y Jarpa-Arriagada

Las experiencias mundiales de cuidado infantil dirigidas a niños/as que no pueden permanecer con su familia, no han mostrado resultados consistentes con la lógica de la protección de derechos. En efecto, diversas investigaciones muestran que cuando un niño/a se institucionaliza puede estar sometido a cambio constante de la figura cuidadora, experiencias de trauma por el abandono o negligencia de sus padres e incluso ser sometido a maltrato infantil en la misma institución que debería protegerlo (Gabatz, Schwartz, Milbrath, Carvalho \& Lange, 2018; García-Quiroga \& Hamilton-Giachritsis, 2017; Hawk et al., 2018; Katsurada, Tanimukai \& Akazawa, 2017; Lino, Nobre-Lima \& Mónico, 2016; MacKenzie et al., 2017; Turney \& Wildeman, 2017).

Una responsabilidad central en las residencias de protección recae en el cuidador o cuidadora. En Chile, este cuidador es un funcionario que no dispone de especialización o formación en el área; tiene un contrato precario en términos de jornada laboral e ingresos y no dispone de mayor experiencia en crianza de niños, salvo su propio ejercicio como padre o madre, cuando llegan a tener dicha condición. El trato de los niños con el cuidador tiene mucho de coloquial. Se le denomina "tío/a", apelativo que es de uso indiscriminado y transversal a todos los dispositivos de cuidado infantil en Chile.

\section{Metodología}

Las representaciones sociales constituyen el enfoque epistemológico y metodológico del estudio. Específicamente, el método utilizado fue el Análisis Estructural del Discurso. Este método resulta útil para aproximarse a las representaciones sociales puesto que establece un encuadre para develar la organización discursiva de los sujetos, los lugares de enunciación, las valoraciones que denota y los significados que se construyen (OpazoValenzuela y Jarpa-Arriagada, 2018). Entendemos las representaciones sociales como sistemas de referencia de los sujetos, que organizan la manera como éstos se explican hechos y relaciones y otorgan sentido, congruencia y dirección a sus actos (Martinic-Valencia, 1992, 2006). Por tanto, el análisis estructural del discurso nos ha permitido identificar unidades básicas de significado en la narración de las y los cuidadores (denominadas códigos), y las respectivas relaciones existentes entre éstos. Las estructuras cruzadas resultantes nos facilitan la comprensión de las acciones realizadas por los cuidadores para el ejercicio de la autoridad y las interacciones significativas en un sistema residencial en Chile.

La investigación se realizó con diez adultos que cumplen función de cuidado en instituciones colaboradoras del Servicio Nacional de Menores (SENAME) en la Región de Nuble, Chile. El perfil de los cuidadores cumplía con un criterio de inclusión principal, cual era acreditar una antigüedad de mínimo dos años en el ejercicio de esta labor. En suma, los/as sujetos/as de investigación tenían un promedio de experiencia de ocho años y sus edades oscilaban entre los treinta y los cincuenta años. 
La entrevista semiestructurada fue la técnica para la producción de datos. Consideró los siguientes tópicos (véase Tabla 1):

Tabla 1. Tópicos de la Entrevista Semi-estructurada.

\begin{tabular}{ll}
\hline Tópico & Sub-tópicos \\
\hline Estilos de autoridad ejercidos & Estilo autorizativo \\
por los cuidadores & Estilo autoritario \\
& Estilo negligente \\
& Estilo indulgente \\
\hline Estilos de interacción con los & Predominancia de límites \\
niños, niñas y adolescentes & difusos \\
& Predominancia de límites \\
& rígidos \\
& Predominancia de límites claros \\
\hline
\end{tabular}

Fuente: elaboración propia.

Respecto de los sub-tópicos, las definiciones de estilo de autoridad corresponden a los autores Musitu-Ochoa y Cava-Caballero (2001), en tanto las definiciones de los límites corresponden a Minuchin citado en Gracia-Fuster, E., y Musitu-Ochoa (2000).

Las definiciones indican:

- Estilo de autoridad autorizativo: corresponde a una relación basada en alta implicación y alta coerción.

- Estilo de autoridad autoritario: corresponde a una relación basada en baja implicación y alta coerción.

- Estilo de autoridad negligente: corresponde a una relación basada en baja implicación y baja coerción.

- Estilo de autoridad indulgente: corresponde a una relación basada en alta implicación y baja coerción.

- Predominancia de límites difusos: corresponde a una relación dónde el límite es excesivamente abierto, permitiendo la intrusión entre individuos y la laxitud de las normas. 
Gallegos-Fuentes y Jarpa-Arriagada

- Predominancia de límites rígidos: corresponde a una relación dónde el límite es excesivamente cerrado, facilitando la autonomía de los individuos y la baja identidad en la relación.

- Predominancia de límites claros: corresponde a una relación dónde el límite está bien definido, permitiendo adecuada permeabilidad y la equilibrada definición de funciones de los participantes en la interacción.

Reconocemos cuatro fases en el desarrollo del estudio: (i) reunión formal con las instituciones residenciales para dar cuenta de la investigación y sus objetivos; (ii) firma del consentimiento informado por parte de los sujetos de la investigación; (iii) producción de datos y análisis de los mismos; (iv) socialización de los hallazgos a los participantes y feedback de parte de éstos.

La confidencialidad se resguardó mediante el anonimato de los sujetos que participaron de la investigación; para ello se utilizan códigos que identifican cada entrevistado. La voluntariedad se respetó en todo el proceso de investigación, esto es, al inicio con la firma del consentimiento y luego con la posibilidad permanente de que cada sujeto tomara la decisión de retirarse si así lo estimaba. Con todo, el resultado del proceso es fruto de una dedicación intencionada por generar una relación dialogante, respetuosa y comprometida tanto con los sujetos como con el estudio realizado.

\section{Hallazgos}

Para aproximarnos al estilo de crianza de los cuidadores, hemos asumido una categorización atribuida a figuras parentales, toda vez que nuestra investigación ha develado que los cuidadores representan un ejercicio de autoridad (Gallegos-Fuentes, JarpaArriagada y Opazo Vega, 2018). Asumimos que el estilo de autoridad transita desde una mayor o menor implicación, en combinación con una mayor o menor coerción. Esto da lugar a cuatro estilos. Autoritario, con dosis altas de coerción y baja implicación; Autorizativo, con alta coerción y alta implicación; Negligente, con baja coerción y baja implicación; e Indulgente, con alta implicación y baja coerción (Musitu-Ochoa y Cava-Caballero, 2001). En el análisis de las relaciones hemos considerado la noción del conflicto, a partir de las habilidades de colaboración, transacción, evasión, adaptación y competencia (Thomas y Kilmann, 2015).

Los resultados obtenidos mediante el Análisis Estructural del Discurso se presentan a través de dos estructuras cruzadas (véase Figura 1 y 2). Cada estructura cruzada define cuatro variaciones posibles y se concretan, cada una, en una categoría específica. Cada una de ellas representan una valoración positiva (+) o negativa (-), develando la posición de los sujetos participantes respecto de lo enunciado. 


\subsection{Estilos de autoridad según los/as cuidadores/as}

Figura 1. Estructura cruzada estilos de autoridad.

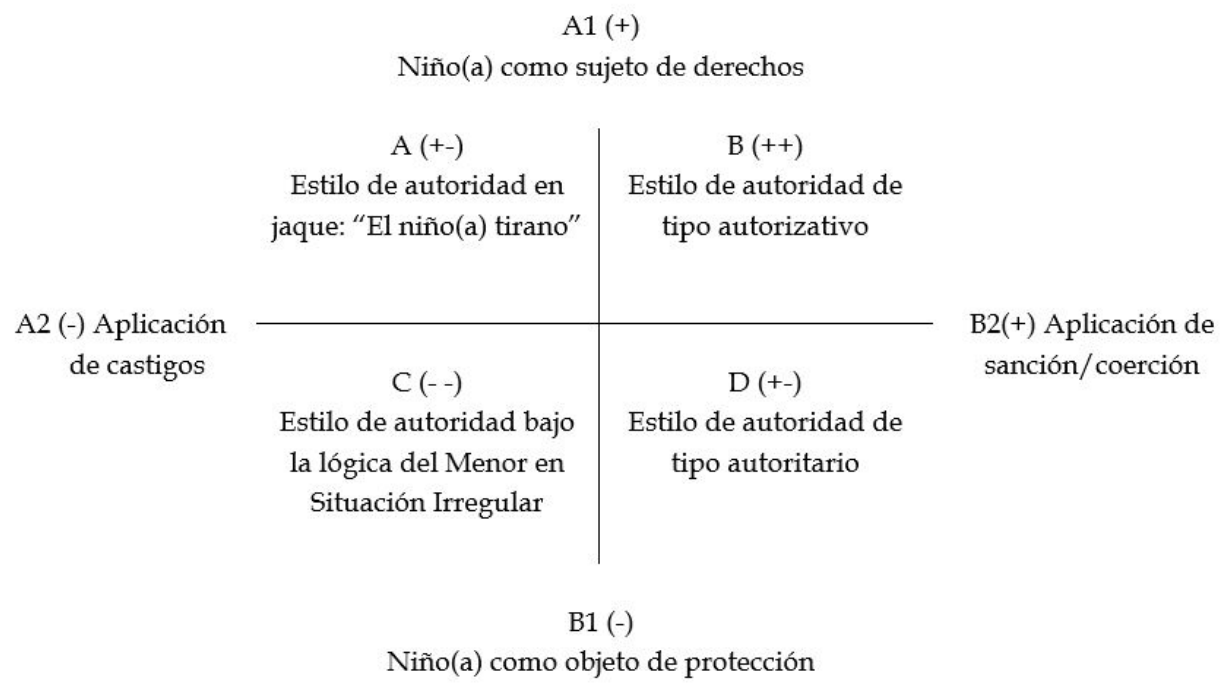

Fuente: elaboración propia.

\section{Estilo de autoridad en jaque "El/La niño(a) tirano/a"}

Desde la representación social de los/as cuidador/as, el/a niño/a tirano/a se configura en una especie de ciclo de conductas desafiantes a la autoridad; este proceso se despliega desde la exigencia de sus derechos, pasando por la amenaza de acusarlos de conductas mal-tratantes, hasta la posibilidad de agresión física dirigida al cuidador. Esta conducta agresiva y activa se gatillaría cuando el niño percibe que el cuidador no cumple sus demandas y, al mismo tiempo, no cuenta con una base de apoyo institucional. Al respecto, la falta de respaldo se advierte desde la frágil posición del cuidador en un entramado institucional que declara la plena adherencia a los derechos del niño. Desde los discursos, la fragilidad del cuidador se articula por cuestiones no resueltas a nivel relacional entre educador y niño. Específicamente, el manejo de las sanciones es un aspecto relacional que abre un flanco para la coherencia del ejercicio del rol del cuidador, en el sentido que dificulta la claridad del rol y su consistencia frente a las demandas de los niños.

...los niños a nosotros nos suben, nos bajan y uno queda... claro más que decirles que no se hace, explicarles que está mal, que es una falta de respeto, pero el niño más allá no, o a veces uno está expuesto a que incluso el niño te pegue, pero uno no tiene una protección sobre eso... 
...aquí ha habido compañeras que han sido maltratadas en forma seria, con piedrazos, que han tenido que ir a la mutual con licencia, entonces es preocupante... no cambian de conducta (los niños) ... entonces los derechos de nosotros están poco claros, pero los derechos de ellos sí... (E3)

Este estilo de autoridad se puede entender en el contexto de una relación de cuidado ejercida en residencias de protección. Los/as cuidadores/as afirman que su autoridad se ve deslegitimada por una lógica de superioridad del niño/a en el vínculo con el adulto, donde los/as cuidadores/as observan una preeminencia de los derechos por sobre los deberes (Gallegos-Fuentes et al., 2018). Ergo, se presentan episodios de abierta subversión del orden jerárquico esperado, lo que concluye con maniobras interaccionales donde el niño/a se sitúa en una posición superior al cuidador/a. El supuesto que alimenta esta perturbación jerárquica es que los/as cuidadores/as no pueden aplicar medidas de control de comportamiento que estén reñidas con sus derechos o al límite de implicar una vulneración de sus derechos. Una pregunta inevitable es ¿qué hace que los cuidadores no apliquen medidas de control no reñidas con los derechos del niño/a?; nuestra hipótesis es que el cuidador/a carece de una formación suficiente en técnicas o herramientas de control conductual que produzcan aprendizajes significativos y no sólo aversión. Para BarreraValencia (2010), una posible explicación sería la discontinuidad cultural que configura trayectorias disímiles entre el adulto y el niño por sus ciclos de vida históricos y los modelos de crianza al que fueron expuestos. En consecuencia, los adultos cuidadores habrían participado de una cultura post figurativa donde los niños aprenden esencialmente de sus mayores, en tanto los niños hoy estarían más expuestos a una cultura pre figurativa donde tienen un protagonismo en los procesos y una influencia más activa respecto de lo que se puede o no se puede hacer en relaciones de autoridad (Barrera-Valencia, 2010).

Ciertamente, si el estilo de autoridad ejercido por los/as cuidadores/as es una coconstrucción, la danza interaccional entre adulto y niño/a produce intercambios que podrían acercarse a un tipo de "regla secreta". En este patrón, los intentos del cuidador/a para ejercer coerción sobre el niño, ante una conducta designada como inapropiada, vienen seguidos de las maniobras del niño/a para deslegitimar este intento, debido a que se encuentra en una residencia de protección infantil y lo asimila a una forma de mal-trato, derivando en una retirada del adulto cuidador en su intento de poner límites, produciendo un debilitamiento de su autoridad y una alteración en la jerarquía niño/adulto. En suma, y tal como plantean Sorkhabi \& Middaugh (2014), la disminución de la legitimidad de la autoridad de los adultos se relaciona con los intentos de los niños de aumentar sus libertades personales al reducir la cantidad de control en diferentes áreas o dominios de sus vidas. 


\section{Estilo de autoridad de tipo autorizativo}

El estilo de autoridad autorizativo forma parte del relato como un ideal, pero con mínima concreción en sus prácticas cotidianas. La imposibilidad de ejercer un estilo autorizativo pasaría por la débil legitimidad que los/as niños/as le atribuyen a la autoridad del adulto a cargo. Por consiguiente, la consecuencia interaccional es la negativa a aceptar el control ejercido por el cuidador (Darling, Cumsille y Martínez, 2008). De esta manera, si los niños no creen que el cuidador puede ejercer control sobre ellos, la legitimidad de la autoridad decae severamente.

Yo lo veo por el lado positivo, porque ya un niño es consciente de lo que le falta... él está aprendiendo poco a poco como debe ser con las demás personas, lo que se merece cada sujeto en su vida... entonces no puedo decir que no me gustaría que estuvieran sumisos y que siempre acataran lo que uno les diera... o sea es necesario que estén conscientes... me parece bien, pero no olvidando que tienen responsabilidades... (E5)

Se le llama sanción, por ejemplo, si un niño se porta mal, antiguamente no había como una sanción, ... ahora se implementó el tema de la sala de computación, que se ha ido mejorando... eso era como lo que ellos buscaban (refiriéndose a los cuidadores), un mejoramiento y ahora eso ha dado resultados... el tema es que si tú te comportas mal, hoy día no entras a computación, cosas así... como que ellos no estaban muy de acuerdo de que antes no había sanción antiguamente... en esos temas... (E2)

Desde la representación de los cuidadores, el ejercicio de un estilo autorizativo requiere de la participación del niño/a como sujeto co-constructor de la alteridad. Un elemento central en esta interacción sería la asunción de sus deberes, cuestión que se ve disminuida en el contexto de una residencia de protección infantil, ya que se caracteriza por la tensión y el conflicto. Nuestra lectura es que existe un diálogo no resuelto respecto del ejercicio de los derechos entre los niños y los adultos, lo que pareciera ser conflictivo porque los vasos comunicantes entre los derechos del niño como espacio declarativo y los derechos que emergen en el espacio cotidiano de una residencia se tensionan por el tránsito entre el paradigma del menor en situación irregular y el niño como sujeto de derechos. Por tanto, coexisten imágenes de los niños como objeto de protección con consistentes representaciones del niño como sujeto de derechos, que deriva en un patrón relacional oscilante entre la lógica proteccional y la lógica de promoción de derechos (Gallegos-Fuentes et al., 2018).

Por otro lado, están las herramientas del ejercicio de la autoridad que despliegan los cuidadores. Al respecto, los cuidadores han ido en búsqueda de técnicas de control conductual basadas en un paradigma más cercano a lo cognitivo-conductual (MonsalveRobayo, Mora-Caro, Ramírez-López, Rozo-Hernández y Rojas-Puerto, 2017). De esta manera, buscan reforzar negativamente las conductas inapropiadas y reforzar 
Gallegos-Fuentes y Jarpa-Arriagada

positivamente las conductas esperadas. No obstante, la narrativa más densa enuncia que los mecanismos de control conductual son acotados e insuficientes, ante habituales conductas desafiantes.

Finalmente, otro elemento a tomar en cuenta es la representación misma del niño y cómo esta influye en la definición del estilo de autoridad. Ciertamente, la pre-configuración del niño como un menor en situación irregular, justifica la inexistencia de medidas correctivas desde dos supuestos. Primero, la imagen de un niño carenciado y vulnerado logra la autocensura del cuidador respecto de la aplicación de medidas connotadas como coercitivas; en segundo término, hay una representación del niño/a como un sujeto que dispone de reducida capacidad de cambio debido a su propia historia vital y las condicionantes socioculturales de su crianza inicial (March, 2007).

....los chiquillos están exigiendo mucho sus derechos, pero están dejando de lado sus deberes...... ellos de repente contestan súper mal a los educadores... los niños que están llegando ahora, y eso se entiende porque vienen de una familia que están acostumbrados a gritar... a tirarse golpes... que el papá le pega a la mamá, donde el hombre es visto como el macho de la casa... por lo tanto, yo pego a las mujeres..., o sea yo entiendo que es por eso que se está dando... pero ellos están más buenos para discutir... más buenos para sacar esa rabia que tienen... (E1)

...ellos dicen tía... nosotros se nos olvidan los deberes, nosotros no podemos respetar... nosotros venimos de un lugar totalmente sin educación, a nosotros no nos van a hacer cambiar nunca jamás, porque nosotros estamos acá... a nosotros nos enseñan a sentarnos bien, a respetar a todo el mundo, pero me voy al lugar de donde yo soy, yo me olvido de todo... (E4)

El discurso de los cuidadores enuncia una visión poco optimista respecto de la posibilidad de aplicar un estilo autorizativo y, consecuentemente, plantean que los niños no aceptan el control ejercido por ellos, pero sí demandan con gran energía que les prodiguen conductas de alta implicación. Esta creencia se sostiene sobre la imagen de niños provenientes de familias que han ejercido un estilo de crianza negligente o indulgente. En consecuencia, los cuidadores enfrentan un obstáculo relevante para el ejercicio de un estilo más dialogante, toda vez que los propios niños afirman la imposibilidad de cambiar sus conductas. Todo lo anterior, nos lleva al fenómeno de los límites en el proceso de crianza; para el caso de los niños en residencia estos límites pueden transitar entre una gran rigidez o una alta difusión en sus familias de origen. En este sentido, podemos extrapolar los hallazgos de varias investigaciones que indican que el tipo de estilo parental influye directamente en los niños/as; en efecto, los estilos de autoridad que se acercan más a la negligencia y a la agresividad se relacionan más con conductas agresivas y desafiantes por parte de los niños respecto de la figura de autoridad (Franco-Nerín, Pérez-Nieto y De DiosPérez, 2014; Monsalve-Robayo et al., 2017; Morales-Chainé, Félix-Romero, Rosas-Peña, López-Cervantes y Nieto-Gutiérrez, 2015). 
De este modo, parece plausible que las conductas desafiantes de los niños sí tengan relación con las prácticas de crianza inicial, pero, por otro lado, si los cuidadores aplicaran un control de mejor calidad, esto es, con una disposición a fijar reglas explícitas, a monitorear su cumplimiento y a gestionar su incumplimiento, las relaciones adulto-niño podrían mejorar sustancialmente (Sorkhabi \& Middaugh, 2014).

\section{Estilo de autoridad bajo la lógica del Menor en Situación Irregular}

El ejercicio de este tipo de autoridad se sostiene en una representación del niño como un sujeto vulnerable y carenciado. En este sentido, existe una dualidad entre la promoción de derechos y la imagen de un niño carenciado. La relación adulto-niño se pre-configura considerando la historia vital del niño lo que, a juicio de los cuidadores, impediría gran parte del proceso formativo. En efecto, el peso de la crianza inicial y los patrones ligados a la violencia, la negligencia o a la indulgencia, no podrían ser resueltos por el trabajo del cuidador, por tanto, se inclinan al desarrollo de acciones de cuidado tradicionales ligadas a la satisfacción de necesidades básicas. Por otra parte, afirmamos que la decisión de los cuidadores de no ejercer un estilo de autoridad firme se asocia a la configuración institucional a partir del paradigma de promoción de derechos y también a la imagen de niño carenciado y sin futuro. Esto define, además, un tipo de relación basada en la lógica de menor en situación irregular.

Yo encuentro que aquí se les da todo, se les da como todo a los chiquillos, lo que más se puede en la residencia... que la educación, vestirse, alimentarse, vivir cómodo... calientito, no encuentro que...como que se les esté faltando... no sé...yo creo... (E2)

... entonces lo más cercano, que tengan una cama calentita, su leche, a que se les escuche, a decirles como te fue hoy día, hacerles un cariñito, cosas así... irlos a buscar al colegio, que es lo más cercano a tener una familia... (E1)

La posibilidad de que los cuidadores en residencias infantiles puedan ejercer todavía un estilo de autoridad vinculado a la doctrina del Menor en Situación Irregular se sustenta sobre la tensión entre cuidar y formar. En efecto, si formar o educar requiere necesariamente de un despliegue equilibrado de afecto y de control, pero en el espacio de las residencias los niños rechazan el control, por entenderlo como un ejercicio ilegítimo de parte del cuidador, lo que "queda" es prodigar cuidados de forma casi exclusiva. Al centrarse sólo en cuidar, se podría hipotetizar una representación asociada a la doctrina del menor en situación irregular y a la prevalencia de un sistema de cuidados bajo la idea de un niño vulnerable e incapaz. En este sentido, aún observamos una creencia proveniente de los propios cuidadores donde su rol es proteger al niño para resguardar sus derechos con una clara tendencia a establecer una conservadora relación asistencial (Gallegos-Fuentes et al., 2018). 


\section{Estilo de autoridad de tipo autoritario}

El estilo autoritario es connotado muy negativamente. Los relatos donde los cuidadores enuncian este estilo, describen cómo ha cambiado el modelo de autoridad predominante sobre los niños, más que intentar reivindicarlo. No obstante lo anterior, los/as cuidadores/as afirman que el niño tiene un conocimiento excesivo de sus derechos en contraposición a un muy débil cumplimiento de sus responsabilidades y eso no ocurría con la predominancia de un modelo más autoritario. Por tanto, junto con la tiranía del niño/a ya descrita como estilo de autoridad, los cuidadores afirman estar en presencia de una alteridad desequilibrada, con tendencia a la manipulación de parte de los niños cuando se aplican medidas de control de comportamiento. En suma, el exagerado predominio de los derechos dejaría al adulto chantajeado en esta nueva construcción de sentido, lo que además sería concordante con otros estudios realizados al respecto (Durán-Strauch, 2017; Gallegos-Fuentes et al., 2018).

\footnotetext{
... a los niños acá de repente se le ponen normas o reglas, pero ellos encuentran que son muy blandas (refiriéndose a los cuidadores) ... a la hora de imponer por ejemplo una sanción, que acá ya no se le llama castigo... la palabra castigo no existe... (E2)

...les han dado mucho al niño... que no hay que retarlo, no hay que castigarlo, se supone que brutalmente no, pero... igual hay que tener su sistema rígido con ellos. (E4)
}

La variabilidad discursiva de los cuidadores respecto del estilo autoritario muestra tres grandes matices. Por una parte, los cuidadores reconocen que el estilo autoritario está censurado como mecanismo de control en el contexto de una residencia de protección infantil. Esta representación se nutre del nuevo encuadre que originó la Convención de los Derechos del Niño y que se materializa en el Paradigma de Protección de Derechos. Esto genera una tensión con la predominancia del lenguaje garantista, un vuelco hacia los derechos y un adulto cuidador chantajeado en este nuevo tipo de relación, dónde el niño define un nuevo vínculo a partir de la predominancia de sus derechos (Gallegos-Fuentes et al., 2018).

Por otra parte, existe una representación anclada a la propia experiencia familiar de los cuidadores que devela que el estilo autoritario sí funciona, siempre que sea "bien utilizado". Nuestro supuesto es que los cuidadores se refieren a su propia vivencia infantil y, también, a su proyecto actual de familia. Esto es, el discurso indicaría que fueron educados bajo un estilo autoritario y dicha crianza contribuyó a su desarrollo como persona. Luego, trasladan este patrón de interacción con sus propios hijos, como una forma de reproducción del estilo de autoridad de sus propios padres. 
A partir de esta experiencia vital, emerge el tercer matiz. Surge la añoranza del estilo autoritario, pero no pueden extrapolarlo a su desempeño como cuidador. En este sentido, la supremacía del niño en la interacción condiciona una fragilidad y oscilación en las decisiones que los cuidadores adoptan respecto del ejercicio genuino de un estilo autoritario, derivando en una inclinación por un estilo más vinculado al menor en situación irregular. En este caso, podemos afirmar la vigencia de un modelo adulto céntrico que no puede expresarse en una relación de poder coercitiva (Zuluaga-Gómez, 2018), pero que encuentra expresión en una relación de protección basada el paradigma del Menor en Situación Irregular.

\subsection{Tipos de interacción cuidador(a)-niño(a) en el contexto residencial}

Figura 2. Estructura cruzada tipos de interacción.

\section{$\mathrm{A} 1(+)$}

Cuidador (a) implicado con los derechos del niño

\begin{tabular}{|c|c|c|c|}
\hline & $\mathrm{A}(+-)$ & $\mathrm{B}(++)$ & \\
\hline & Relación & Relación & \\
\hline & conflictiva/divergente & Nutritiva/convergente & ה"ח \\
\hline ejerce derechos & & & ejerce derechos \\
\hline & $C(--)$ & $\mathrm{D}(+-)$ & \\
\hline instrumental & & & legítima \\
\hline & Conflictiva/indulgente & Conflictiva/negligente & \\
\hline
\end{tabular}

B1 (-)

Cuidador(a) apático con los derechos del niño

Fuente: elaboración propia.

Respecto de las categorías propuestas, los hallazgos sólo validan tres de ellas, esto es, la relación conflictiva divergente, la conflictiva negligente y la nutritiva convergente. 


\section{Interacción de tipo conflictiva divergente}

En esta categoría se reúnen enunciados de los cuidadores que develan interacciones donde el conflicto surge por discrepancias frecuentes de la vida cotidiana y que traspasan las expectativas de armonía interpersonal entre cuidador/a y niño/a. Si entendemos el conflicto como oposición mutua y expresión manifiesta de diferencias entre dos o más individuos (Shantz \& Hartup, 1992), la conducta de antagonismo entre cuidador/a y niño/a puede tener múltiples expresiones, pero el campo más habitual de manifestación son las rutinas de la vida cotidiana.

El contexto relacional en el que ocurren los conflictos puede ser un factor importante para determinar en qué medida los conflictos son interacciones funcionales o educativas (Sorkhabi \& Middaugh, 2014); en el marco de una institución de protección y desde los discursos de los/as cuidadores/as, los conflictos parecen responder más a una lógica funcional y establecerse como una forma esencial de comunicación (Adams \& Laursen, 2007).

...los niños a nosotros nos suben, nos bajan y uno queda... claro más que decirles que no se hace, explicarles que está mal, que es una falta de respeto, pero el niño más allá no, o a veces uno está expuesto a que, incluso el niño te pegue, pero uno no tiene una protección sobre eso... (E7)

Sí... porque si uno les pide... ellos no obedecen y se alteran... entonces siempre hay que ver cómo andan ellos... por ejemplo... ya haz la cama... su deber es hacer la cama cierto... y dicen no yo no quiero, no quiero... se enojan y al final uno no sabe cómo hacerlo... (E4)

El discurso de los cuidadores muestra que el tipo de interacción se relaciona de manera coherente con el estilo de autoridad ejercido. En efecto, existe sintonía entre el Estilo de autoridad en jaque (El niño tirano) con la interacción conflictiva divergente. Por tanto, los antagonismos cotidianos dan como resultado la ejecución de acciones de control por parte del cuidador que colisionan con las manipulaciones del niño; todo lo anterior provoca una retirada del cuidador y una discordancia relacional para la búsqueda de soluciones en un esquema de promoción de derechos. Ciertamente, cuando el cuidador decide alejarse lo hace como un mecanismo de autoprotección ante eventuales acusaciones de mal-trato; por su parte, el niño responde al alejamiento situándose en una jerarquía superior, provocando una perturbación de los límites. Lo paradojal es que la interacción conflictiva y divergente se expresa en un contexto de protección de derechos, pero que, contrariamente, logra materializarse en una danza interaccional que redunda en el cumplimiento errático del ejercicio de derechos, en beneficio de la homeostasis. 


\section{Interacción de tipo nutritiva convergente}

Esta categoría reúne enunciados que ilustran un tipo de interacción dónde se logra sinergia entre cuidador y niño, en dirección al desarrollo del niño como meta principal. Lo nutritivo lo entendemos desde Satir (2004), dónde una relación adulto/niño provee autoestima positiva, define reglas claras y compartidas y prima un tipo de comunicación que proporciona seguridad y estabilidad. La convergencia permite vivenciar una sensación de proyecto conjunto, de caminos confluentes y de una mayor cohesión.

Aparentemente, este tipo de interacción podría alimentar con más solidez un estilo autorizativo, pero su densidad discursiva es baja y queda debilitada desde un "discurso híbrido" (Durán-Strauch, 2017), que acopla armoniosamente la asistencialidad con los derechos del niño. La complejidad de la relación de cuidado se sobrelleva con una alta dosis de expresiones de afecto y un sentido del cumplimiento del deber (Gallegos-Fuentes et al., 2018); de este modo, la expresión de sentimientos, la contención afectiva y la protección son valoradas de forma más significativa que la independencia de los niños (García-Quiroga \& Hamilton-Giachritsis, 2017).

uno tiene que tener una buena acogida con el niño... expresarle cariño al momento de recibirlo, mostrarle los compañeros que va a tener, mostrarle su camita, en donde va a dormir... dar las pautas... los horarios de levantada... de almuerzo, colación, cena... que en la mañana cada uno hace sus quehaceres en la casa... (E6)

....reírnos con un chico, ...conversamos en la mesa y... no hay discusiones...si uno los va a dejar... ir a dejarlos, decirles chao, darles un beso, que les vaya bien. (E1)

Cuando impera este campo semántico, los cuidadores se acercan a su rol de protección con mayor comodidad. Consecuentemente, las "prácticas de cuidado" develan significados de provisión y protección, que se armonizan en torno al propósito de formar niños en desarrollo y que contribuyen significativamente a la identidad del cuidador por tratarse de niños/as que viven vulneraciones de sus derechos. En esta representación, se advierte una semántica que sitúa al niño no sólo como alguien que requiere de una plena satisfacción de sus necesidades básicas, sino como alguien que debe ser visto más integralmente, en una mirada de plena alteridad (Gallegos-Fuentes et al., 2018).

\section{Interacción de tipo conflictiva negligente}

Esta categoría se presenta con baja densidad. Sin embargo, nos parece interesante discutir algunas implicaciones que un discurso como este podría plantear en el contexto de nuestra investigación. Cuando hablamos de una interacción conflictiva negligente nos referimos a un tipo de interacción dónde el adulto responsable muestra muy baja implicación y muy baja coerción en su relación con el niño. La ausencia o bajísima presencia 
de implicación y coerción suscitaría el conflicto, en la medida que resulta casi impensable que un adulto que está contratado para cumplir funciones de cuidado, no las realice.

Pero ella siempre lo trató con amor, que hijo para acá, que hijo para allá, pero había otra tía que le faltaba un poco el respeto y ya le hacían como la cruz al niño y que tampoco es la idea ... (E1) Allá donde estaba yo antes, al niño si no funcionaba en la casa, la mamá no lo tomaba más en cuenta, lo cambiaban a otra casa y empezar de cero... (E1)

Este preliminar hallazgo, desnudaría un tipo de relación que no es reconocida en la vivencia de los cuidadores, pero que sí se reconoce en "otros", casi como una suerte de proyección. Posiblemente, la deseabilidad del discurso actúa como un filtro que impide el reconocimiento de conductas como esta, por estar severamente reñidas con su rol de cuidadores. En este sentido, el campo semántico parece congruente en cuanto a la negativa de actuaciones autoritarias y negligentes en la relación con los niños, ya que ambas son abiertamente contradictorias con su mandato laboral.

\section{Conclusiones}

Para la presentación de las consideraciones finales hemos construido una última estructura cruzada que agrupa los resultados más relevantes de nuestro estudio. Como se puede apreciar en la Figura 3, es posible reconocer cuatro Representaciones Sociales que agrupan universos semánticos sobre el estilo de autoridad e interacción que los cuidadores ejercen con los NNA.

Para efectos de la comprensión de estas representaciones, es preciso puntualizar que no se trata de compartimentos estancos. Más bien, operan muy interconectadamente unas con otras, en una especie de bucle, con idas y vueltas. Posiblemente, los énfasis van a estar relacionados con las vicisitudes peculiares que el cuidador/a enfrenta en la vida cotidiana de la residencia. El principal hallazgo es que las representaciones no se anulan entre sí. Son co-existentes, iterativas, interconectadas y, muchas veces, confusas. Resulta paradojal que, en tanto predominó hegemónicamente la Doctrina del Menor en Situación Irregular, la actuación de los cuidadores tenía absoluta certeza. En el dominio de la nueva Doctrina de Protección Integral se advierten flancos abiertos, que generan incertidumbre e inestabilidad.

De esta manera, los estilos de autoridad de los cuidadores/as no son ni permanentes ni estables. Al contrario, son oscilantes, y están definidos por el tipo de relación que se configura en situaciones determinadas o particulares. Más bien, los relatos sugieren que existe una adecuación a las distintas circunstancias que enfrentan. Esto significa que, dependiendo del contexto, pueden establecer relaciones desde un estilo de autoridad autorizativo y otras desde un estilo diferente. 
Gallegos-Fuentes y Jarpa-Arriagada

\section{Representación Social de la relación cuidador/a-niño/a según los/as cuidadores/as}

Figura 3. Estructura cruzada representación social cuidadores/as.

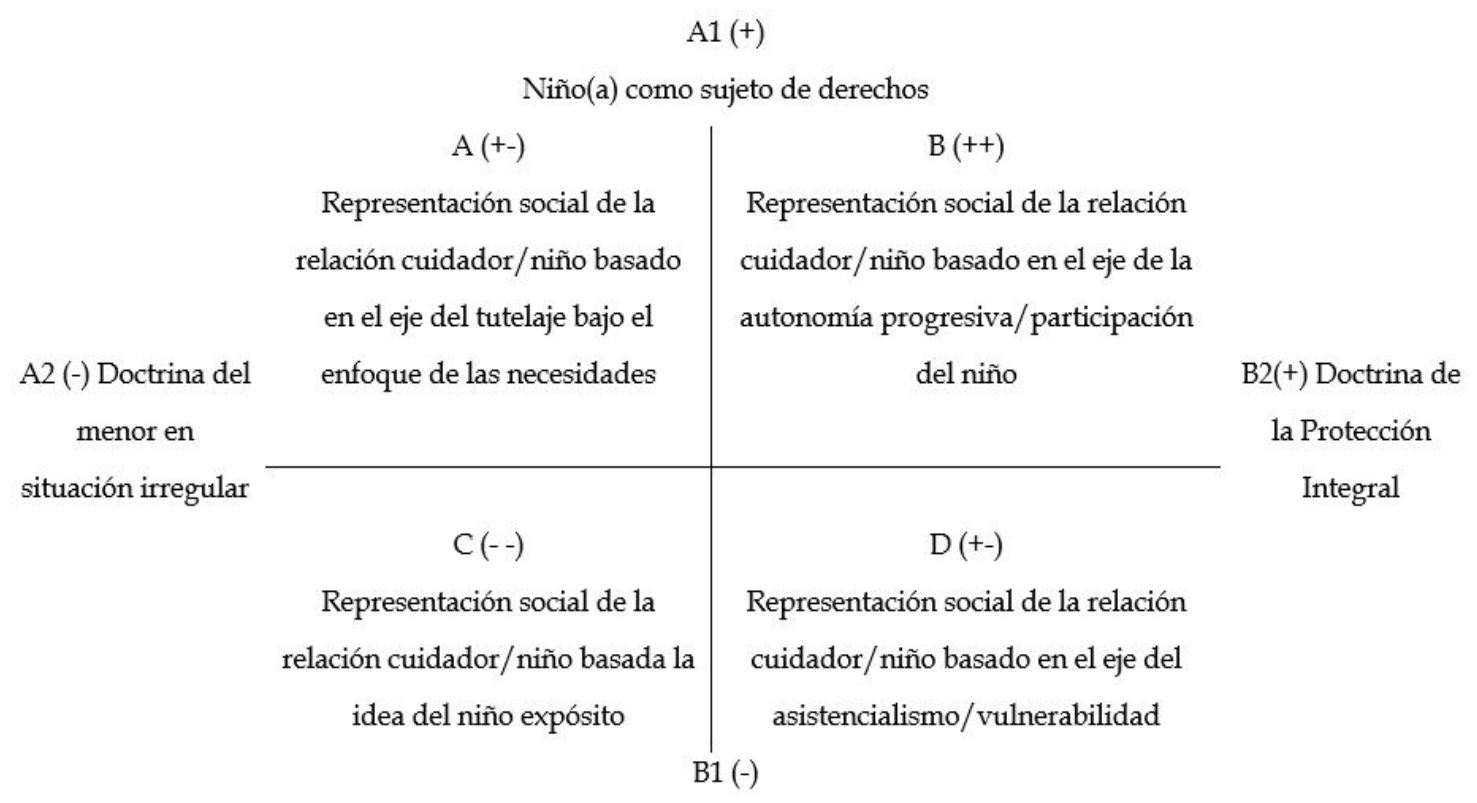

Niño(a) como objeto de protección

Fuente: elaboración propia.

Representación social de la relación cuidador/niño basado en el eje de la autonomía progresiva/participación

Este núcleo figurativo reúne concepciones idealizadas de las posibles interacciones cuidador/a-niño/a. La posibilidad de construir relaciones que se sostengan sobre la doctrina de la protección integral y que promuevan, de manera genuina, los derechos de los niños supone a lo menos dos requisitos: (i) un contexto institucional que establezca un encuadre claro y explícito para los cuidadores y (ii) cuotas de mayor implicación del cuidador en la relación con el niño para favorecer la desestabilización de creencias muy arraigadas, como el determinismo a partir del origen sociocultural de los NNA. 
Representación social de la relación cuidador/niño basado en el eje del asistencialismo/vulnerabilidad

Este núcleo figurativo emerge como una representación contradictoria. Se nutre del tipo de respuesta de los cuidadores frente a la imagen del niño tirano y como alternativa al estilo autorizativo. La resultante es un cuidador que se aferra a la imagen de un niño dañado y que necesita ser protegido. La tiranía del niño es aceptada como una modalidad de evasión de conflictos y una estrategia de autoprotección para no ser acusados de conductas maltratantes. Aunque parezca inusitado, la representación social del asistencialismo/vulnerabilidad puede derivar en un conjunto complejo de conductas que encuentran su explicación en la retirada del cuidador, la baja implicación y el uso de la evasión como estrategia para resolver los conflictos.

En este contexto, emerge una representación dónde el estilo autoritario, junto con el estilo de autoridad bajo la lógica del Menor en Situación Irregular (M.S.I) forman parte del repertorio que el cuidador adopta para relacionarse con los niños y resolver los problemas cotidianos. Ciertamente, frente a la censura por el autoritarismo, aparece el estilo basado en el M.S.I como casi única herramienta sobre la cual se construye la relación. Existe un fuerte arraigo discursivo-semántico en los cuidadores, donde aparece como la única salida, indefectiblemente. No hay reconocimiento explícito de la severa contradicción que se produce con la Doctrina de la Protección Integral, ya que está sólidamente engarzada al discurso del cuidador como herramienta mediadora entre adulto y niño y que, seguramente, podría albergar otras herramientas no reconocidas explícitamente como el castigo. Lo anterior, podría tener relación con la falta de definición respecto del tipo de autoridad adecuada al contexto institucional y a una aún débil adherencia práctica al modelo de promoción de derechos desde la nueva Doctrina de la Protección Integral.

Representación social de la relación cuidador/niño basado en el eje del tutelaje bajo el enfoque de necesidades

Este núcleo reúne una semántica ligada a un enfoque basado en las necesidades (Oyarzún, Dávila, Ghiardo y Habibovic, 2008), dónde la imagen predominante del niño es la de un objeto de protección. El rol de cuidador/a se asocia a un intenso componente filantrópico, coherente con la Doctrina del menor en situación irregular, y adopta una impronta con fuerte connotación asistencial.

Afirmamos que cuando los cuidadores actúan desde esta representación, el nivel de comprensión de la Doctrina de Protección Integral de Derechos no ha logrado una robusta operacionalización de sus principios en la vida cotidiana de la residencia. En consecuencia, advertimos una especie de ejercicio declarativo del paradigma de promoción de derechos que no ha logrado ser resuelto en términos de acciones coherentes. Emergen así las 
contradicciones discursivas que operan a nivel de la acción concreta de los cuidadores, cuya ocupación central continúa inundada por responder a las características de los niños, en una suerte de estigmatización no reconocida.

La nueva matriz comprensiva sobre la co-responsabilidad entre familia, comunidad y Estado para brindar un espacio de desarrollo nutritivo para todos los niños y niñas, no ha entregado claras orientaciones sobre el ejercicio de la supremacía del derecho del niño. Esto es, en una situación de controversia ¿quién define el interés superior?, ¿el niño lo define? o ¿lo define el garante? Por tanto, lo que ocurre en la vida cotidiana de las residencias de protección infantil es que ante cuestiones que deben ser resueltas de manera rápida e inmediata, se opera bajo la lógica del cuidador a cargo. Ciertamente, el cuidador hará uso de las herramientas que están más a su mano, que para el caso de la investigación, es un enfoque más basado en las necesidades del niño, habida consideración de su perfil de niño vulnerable y vulnerado.

\section{Representación social de la relación cuidador/niño basada en la idea del niño expósito}

Este núcleo reúne la semántica de los cuidadores/as respecto de los niños como “hijos del vicio" (Sánchez-Villa, 2016), que era la denominación que asumían los niños que provenían de padres con muchas dificultades. Estas dificultades se expresaban en los niños a través de la herencia y determinaban no sólo sus condiciones biológicas, sino también su conducta social. Esta representación logró validación incluso científica, por lo que no resulta extraño que hasta el día de hoy, los mecanismos de herencia biológica, sean contemplados por adultos cuando se refieren al potencial del niño, su CI, o sus taras.

En esta semántica, entonces, los cuidadores/as, de alguna manera, atribuyen los hándicaps de los niños a su entorno familiar y, específicamente, a su ontogenia. Justifican las conductas de los niños con base a su reproducción sociocultural, incluso las conductas violentas, considerando que no les queda mucho más por hacer porque es una cuestión inmodificable. Lo curioso es que los niños/as también han incorporado este discurso y se definen a sí mismos como alguien que no puede cambiar, o, al menos, que tendrá muchas dificultades para hacerlo porque no logra extrapolar lo que le entregan en la residencia hacia su familia. Allí, no están las condiciones apropiadas. Si nos damos cuenta, esta representación social juega una suerte de desesperanza aprendida no solo en el cuidador, sino que también en el niño, lo que provoca un reforzamiento de las creencias arraigadas en ambos. 


\section{Referencias bibliográficas}

Adams, R., \& Laursen, B. (2007). The correlates of conflict: Disagreement is not necessarily detrimental. Journal of Family Psychology, 21(3), 445-458. doi: 10.1037/08933200.21.3.445.

Barrera-Dávila, S. (2014). De la doctrina de la situación irregular a la doctrina de la protección integral en el Perú. El caso de los hogares del Inabif (Tesis de maestría). Universidad Nacional Mayor de San Marcos. Lima, Perú.

Barrera-Valencia, D. (2010). Empujes y resistencias al cambio en familias con hijos adolescentes. Boletín de Antropología, Universidad de Antioquia, 24(41), 376-394.

Beloff, M. (2000). Los sistemas de responsabilidad penal juvenil en América Latina. Revista Jurídica de la Universidad de Palermo, 5(1), 161-180.

Campos-García, S. (2009). La Convención sobre los Derechos del Niño: El cambio de paradigma y el acceso a la justicia. Revista IIDH, 50, 351-378.

Darling, N., Cumsille, P., \& Martínez, M. L. (2008). Individual Differences in Adolescents' Beliefs About the Legitimacy of Parental Authority and Their Own Obligation to Obey: A Longitudinal Investigation. Child Development, 79(4), 1103-1118.

Durán-Strauch, E. (2017). Derechos de niños y niñas: Del discurso a la política local. Revista Latinoamericana de Ciencias Sociales, Niñez y Juventud, 15(2), 879-891. doi: 10.11600/1692715x.1520623062016.

Farías, A. M. (2003). El difícil camino hacia la construcción del niño como sujeto de derechos. Revista de Derechos del Niño, (2), 187-224.

Franco-Nerín, N., Pérez-Nieto, M. Á., y De Dios-Pérez, M. J. (2014). Relación entre los estilos de crianza parental y el desarrollo de ansiedad y conductas disruptivas en niños de 3 a 6 años. Revista de Psicología Clínica con Niños y Adolescentes, 1(2), 149-156.

Gabatz, R. I. B., Schwartz, E., Milbrath, V. M., Carvalho, H. C. W., \& Lange, C., Soares, M. C. (2018). Formation and disruption of bonds between caregivers and institutionalized children. Revista Brasileira de Enfermagem, 71(supl 6), 2650-2658. doi: 10.1590/00347167-2017-0844.

Gallegos-Fuentes, M., Jarpa-Arriagada, C. G., y Opazo-Vega, D. (2018). Derechos del niño y sistemas residenciales en Chile: Representaciones sociales de adultos cuidadores. Psicoperspectivas. Individuo y Sociedad, 17(2), 1-12. doi: 10.5027/psicoperspectivasVol17-Issue2-fulltext-1184.

García Quiroga, M., \& Hamilton-Giachritsis, C. (2017). "Getting involved": A thematic analysis of caregivers' perspectives in Chilean residential children's homes. Journal of Social and Personal Relationships, 34(3), 356-375. doi: 10.1177/0265407516637838.

García-Méndez, E. (1994). Derecho de la Infancia Adolescencia en América Latina: De la situación irregular a la protección integral. Bogotá: Forum Pacis.

Gracia-Fuster, E., y Musitu-Ochoa, G. (2000). Psicología social de la familia. Buenos Aires, Argentina: Paidos. 
Hawk, B., Mccall, R., Groark, C., Muhamedrahimov, R., Palmov, O., \& Nikiforova, N. (2018). Caregiver Sensitivity and Consistency and children's prior family experience as contexts for early development within institutions: Sensitivity and Consistency in Institutions. Infant Mental Health Journal, 39(4), 432-448. doi: 10.1002/imhj.21721.

Katsurada, E., Tanimukai, M., \& Akazawa, J. (2017). A study of associations among attachment patterns, maltreatment, and behavior problem in institutionalized children in Japan. Child Abuse \& Neglect, 70, 274-282. doi: 10.1016/j.chiabu.2017.06.018.

Lino, A. M., Nobre-Lima, L., \& Mónico, L. S. (2016). The moderating role of length of stay in the relationship between cognitive dysregulation and peer attachment in adolescent boys and girls living in residential care. Children and Youth Services Review, 71, 290-298. doi: 10.1016/j.childyouth.2016.11.018.

MacKenzie, M. J., Schwalbe, C., Gearing, R. E., Ibrahim, R. W., Conway, A., Brewer, K. B., \& Shaker, Z. B. (2017). Dysregulated infant temperament and caregiver warmth in Jordanian orphanages: The importance of considering goodness-of-fit. Children and Youth Services Review, 82, 400-406. doi: 10.1016/j.childyouth.2017.10.012.

Maldonado-Fuentes, F. (2014). Estado y perspectivas de la reforma proyectada en Chile sobre el sistema de protección de menores de edad. Lus et Praxis, 20(2), 209-233.

March, R. (2007). Claves para la intervención con menores acogidos en recursos residenciales, que presentan conductas problemáticas. Intervención Psicosocial, 16(2), 213-227.

Martinic-Valencia, S. (1992). Análisis Estructural: presentación de un método para el estudio de lógicas culturales. Chile: Centro de Investigación y Desarrollo de la Educación (CIDE).

Martinic-Valencia, S. (2006). El estudio de las representaciones y el análisis estructural del discurso. En M. Canales-Ceron (Ed.), Metodologías de investigación social: introducción a los oficios (pp. 299-319). Chile: LOM Ediciones.

Monsalve-Robayo, A., Mora-Caro, L. F., Ramírez-López, L. C., Rozo-Hernández, V., y RojasPuerto, D. M. (2017). Estrategias de intervención dirigidas a niños con trastorno negativista desafiante, una revisión de la literatura. Ciencias de la Salud, 15(1), 105-127. doi: 10.12804/revistas.urosario.edu.co/revsalud/a.5384.

Morales-Chainé, S., Félix-Romero, V., Rosas-Peña, M., López-Cervantes, F., y NietoGutiérrez, J. (2015). Prácticas de crianza asociadas al comportamiento negativista desafiante y de agresión infantil. Avances en Psicología Latinoamericana, 33(1), 57-76. doi: 10.12804/apl33.01.2015.05.

Musitu-Ochoa, G., y Cava-Caballero, M. J. (2001). La familia y la educación. Barcelona, España: Octaedro.

Opazo-Valenzuela, P. A., y Jarpa-Arriagada, C. G. (2018). Identidad profesional: representaciones sociales de trabajadoras sociales chilenas en tiempos de dictadura. Revista Katálysis, 21(1), 168-177. doi: 10.1590/1982-02592018v21n1p168. 
Gallegos-Fuentes y Jarpa-Arriagada

Oyarzún, A., Dávila, O., Ghiardo, F., y Habibovic, F. (Eds.). (2008). ¿Enfoque de derechos o enfoque de necesidades? Modelo de gestión para el desarrollo de un sistema local de protección de derechos de la infancia y adolescencia. Chile: Centro de Estudios Sociales (CIDPA); Servicio Nacional de Menores (SENAME).

Prieto-Cruz, O. (2012). Doctrina de Protección Integral y contexto para el análisis de la población adolescente en condición de calle en Costa Rica. Revista Ciencias Sociales, 4(138), 61-75. doi: 10.15517/ rcs.v0i138.12086.

Sánchez-Villa, M. C. (2016). "Los hijos del vicio". El problema del niño expósito y la modernización de la Inclusa en España durante el cambio de los siglos XIX y XX. Cuadernos de Historia Contemporánea, 38, 325-352. doi: 10.5209/CHCO.54302.

Satir, V. (2004). Relaciones humanas en el núcleo familiar. México: Pax-México.

Shantz, C., \& Hartup, W. (Eds.). (1992). Conflict in child and adolescent development. Cambridge: Cambridge University Press.

Sorkhabi, N., \& Middaugh, E. (2014). How Variations in Parents' Use of Confrontive and Coercive Control Relate to Variations in Parent-Adolescent Conflict, Adolescent Disclosure, and Parental Knowledge: Adolescents' Perspective. Journal of Child and Family Studies, 23(7), 1227-1241. doi: 10.1007/s10826-013-9783-5.

Thomas, K., y Kilmann, R. (2015). TKI. Instrumento Thomas-Kilmann de modos de conflicto. España: Projective Way.

Turney, K., \& Wildeman, C. (2017). Adverse childhood experiences among children placed in and adopted from foster care: Evidence from a nationally representative survey. Child Abuse E Neglect, 64, 117-129. doi: 10.1016/j.chiabu.2016.12.009.

Vincenzino, M. (2017). Sobre el Derecho de Niños y Adolescentes. Subjetividad reguladora versus Ciudadanía emancipadora. Margen. Revista de Trabajo Social y Ciencias Sociales, (87), 1-7.

Zuluaga-Gómez, A. (2018). La recrianza humanizada: Un giro a las relaciones de poder y al paradigma adultocéntrico en las instituciones de protección de niños, niñas y adolescentes en situación de vulneración de derechos. Revista Electrónica Educare, 22(2), 1-14. doi: 10.15359/ ree.22-2.20. 


\section{OTROS ARTÍCULOS DE PROSPECTIVA No. 31 DE 2021}

\section{PRESENTACIÓN}

Presentación. Reflexiones sobre desafios al publicar sistematizaciones

Rosa María Cifuentes-Gil

\section{EDITORIAL}

Reflexiones sobre Trabajo Social: aportes de la Sistematización

María Rocío Cifuentes-Patiño

\section{ARTÍCULOS}

Hacer lo que se sabe, pensar lo que se hace. La sistematización como modalidad investigativa Alfonso Torres-Carrillo

Aportes y desafios de la Sistematización de experiencias en el Trabajo Social y la extensión crítica. Apuntes y reflexiones desde la perspectiva de la Educación Popular

María Rosa Goldar

Valeria Chiavetta

La sistematización en Trabajo Social y la epistemología feminista del punto de vista. Diálogos sobre la producción de conocimiento sustentada en experiencias

Ruth Noemí Parola

María Florencia Linardelli

La Sistematización investigativa de las experiencias: del baile de los que sobran a la fiesta de los que faltan

María Belén Ortega-Senet

Sistematización y Trabajo Social en Chile. El largo y sinuoso camino

Patricia Lorena Castañeda-Meneses

Ana María Salamé-Coulon
Sentipensar la pandemia COVID-19 desde la sistematización de la experiencia en Trabajo Social: reflexiones del profesor Oscar Jara Holliday

Elia Sepúlveda-Hernández

La sistematización de experiencias, una investigación social cualitativa que potencia buenas prácticas de convivencia y gobierno. La experiencia de un conjunto residencial multifamiliar en Cali, Colombia Martha Lucia Echeverry-Velásquez Manuela Prada-Dávila

Construcción de subjetividades epistemológicaspolíticas de profesoras y profesores de Investigación social en una universidad privada y confesional en Bogotá

Giovanni Mora-Lemus

Sistematización de la experiencia Reconocimiento de los derechos humanos del adulto mayor en dos familias residentes en Cali y Valledupar (Colombia)

Lina María Cuello-Lacouture

Jimena del Pilar Jaramillo-Jaramillo

La memoria transformadora como estrategia de intervención profesional en los procesos de reconciliación social: comprensión a partir de mujeres campesinas, excombatientes y jóvenes en Manizales, Colombia

Yeimmy Stephania Corredor-Sotelo

Juliana Fuertes-Fuertes

Sistematización de una estrategia de educación informal implementada en personas privadas de la libertad en el establecimiento penitenciario de mediana seguridad y carcelario de Barranquilla, Colombia

Rafael Humberto Herrera-Mercado Rafael Alberto Zambrano-Vanegas 
Aportes significativos del proceso de intervención comunitaria con la Escuela Popular de Comunicación Alternativa Jaime Garzón de la ciudad de Cúcuta, Colombia

Carlos Lasso-Urbano

La sistematización de la intervención como metodología de investigación en Trabajo Social. Importancia práctica y teórica de la fase de recogida de datos en la intervención social según experiencia del Programa de Apoyo a las Familias en Zaragoza, España

Elisa Esteban-Carbonell

Nuria Del Olmo-Vicén

Papel de la sistematización de experiencias en los procesos de evaluación de intervenciones de salud pública en la Comuna Saludable por la Paz, Cali - Colombia

Jenny Faisury Peña-Varón

Paola Andrea Marín-Velásquez

Janeth Mosquera-Becerra

Experiencia de intervención social en hogares comunitarios integrales del barrio Alfonso Bonilla Aragón, Cali - Colombia

Julián Alexander Montaño-Cárdenas

Las políticas sociales y el gobierno de la "población indígena". Estrategias y regulaciones en el multiculturalismo chileno

Rodrigo Agustín Navarrete-Saavedra

Representaciones sociales sobre estilo de autoridad y tipos de interacción en cuidadores de residencias de protección infantil en Chile

Marcelo Gallegos-Fuentes
Carmen Gloria Jarpa-Arriagada

Reflexiones sobre inseguridad social y cuestiones penales. Una respuesta estratégica a partir de experiencias de cooperativismo con ex detenidos en Argentina

Analia Elizabeth Otero

Yael Yanina Barrera

Desarrollo y salud: la emergencia de un nuevo paradigma

Jesús María Sánchez-Ordóñez

Trabajo Social en ejercicio libre: la perspectiva profesional en España

Paula Frieiro-Padín

Tamara Fernández-Arias

Rubén González-Rodríguez

\section{RESEÑAS}

Social Work and the City: Urban Themes in 21stCentury Social Work

Felipe Saravia-Cortés

Respuestas del Trabajo Social ante emergencias sociales y problemáticas sociales complejas de México y España

Felipe Saravia-Cortés

El feminismo, el género y la profesionalización del trabajo social en Colombia (1936-2004)

Ambar Oriana Serna-Lombo

El puño invisible. Arte, revolución y un siglo de cambios culturales

Carlos Arturo Robledo-Marín

\section{PROSPECTIVA}

No. $31 \bullet$ ene.-jun. 2021

e-ISSN: 2389-993X • Universidad del Valle 